\title{
THE POISSON DISTRIBUTION SERIES OF GENERAL SUBCLASSES OF UNIVALENT FUNCTIONS
}

\author{
S. Porwal, Ş. Altinkaya and S. Yalçin
}

Abstract. The motivation of this paper is to initiate connections between varied subclasses of univalent functions involving the Poisson distribution series.

2010 Mathematics Subject Classification: 30C45.

Keywords: Poisson distribution series, univalent functions, starlike functions, convex functions.

\section{INTRODUCTION}

Let $\Delta$ be the unit disk

$$
\{z \in C:|z|<1\}
$$

and let $A$ be the class of functions analytic in $\Delta$, satisfying the normalization condition $f(0)=f^{\prime}(0)-1=0$. Then each $f \in A$ has the Taylor expansion

$$
f(z)=z+\sum_{n=2}^{\infty} a_{k} z^{k} .
$$

As usual, by $S$ we represent the class of all functions in $A$ which are univalent in $\Delta$.

A function $f \in A$ is said to be starlike of order $\mu$ if it satisfies

$$
\Re\left\{\frac{z f^{\prime}(z)}{f(z)}\right\}>\mu \quad(0 \leq \mu<1, z \in \Delta),
$$

is said to be convex of order $\mu$ if it satisfies

$$
\Re\left\{1+\frac{z f^{\prime \prime}(z)}{f^{\prime}(z)}\right\}>\mu \quad(0 \leq \mu<1, z \in \Delta) .
$$


These classes represented by $S^{*}(\mu)$ and $K(\mu)$, respectively, were first introduced by Robertson [6]. We note that

$$
K(\mu) \subset S^{*}(\mu) \subset A
$$

Let $T$ indicate the subclass of $S$ consisting of functions whose coefficients, from the second on, are non zero given by (see [7])

$$
f(z)=z-\sum_{n=2}^{\infty} a_{k} z^{k} \quad\left(a_{k} \geq 0\right) .
$$

We indicate by $T^{*}(\mu)$ and $C(\mu)$, respectively, the classes obtained by taking the intersections of $S^{*}(\mu)$ and $K(\mu)(0 \leq \mu<1)$ with $T$,

$$
\begin{gathered}
T^{*}(\mu):=S^{*}(\mu) \cap T \\
C(\mu):=K(\mu) \cap T .
\end{gathered}
$$

Definition 1. (See [3]) A function $f \in T$ is said to be in the class $U(\lambda, \alpha, \mu)$, if it satisfies the inequality:

$$
\begin{gathered}
\Re\left(\frac{z \Psi^{\prime}(z)}{\Psi(z)}\right)>\mu \\
(0 \leq \alpha \leq \lambda \leq 1,0 \leq \mu<1, \quad z \in \Delta),
\end{gathered}
$$

where

$$
\Psi(z):=\lambda \alpha z^{2} f^{\prime \prime}(z)+(\lambda-\alpha) z f^{\prime}(z)+(1-\lambda+\alpha) f(z) .
$$

The function class $U(\lambda, \alpha, \mu)$ is of notable interest and it comprises many common classes of univalent functions (see [8]). Further we get [cf. equation (3)]

$$
U(0,0, \mu)=T^{*}(\mu), U(1,0, \mu)=C(\mu) .
$$

By choosing $\mu=0$, we assert the results established by [1], [7].

\section{Preliminary Results}

We employ the tecnique adopted by Porwal [5] to get the Poisson distribution series for univalent functions. 
Just recently, in [5], Porwal establish a power series by making use of the Poisson distribution

$$
\varphi(\xi, z)=z+\sum_{n=2}^{\infty} \frac{e^{-\xi} \xi^{k-1}}{(k-1) !} z^{k} \quad(z \in \Delta) .
$$

In [5], Porwal also define the series

$$
\Omega(\xi, z)=2 z-\varphi(\xi, z)=z-\sum_{n=2}^{\infty} \frac{e^{-\xi} \xi^{k-1}}{(k-1) !} z^{k} \quad(z \in \Delta) .
$$

To demonstrate our first theorem, we express the following Lemma.

Lemma 1. (See [3]) A function $f \in T$ given by (2) is in the class $U(\lambda, \alpha, \mu)$ if and only if

$$
\sum_{n=2}^{\infty}(k-\mu)[(k-1)(k \lambda \alpha+\lambda-\alpha)+1] a_{k} \leq 1-\mu .
$$

Making use of the techniques and methodology used by Porwal [5] (see also [1], [2], [4]), in this present paper, we supply necessary and sufficient conditions for the Poisson distribution series functions belonging to the class $U(\lambda, \alpha, \mu)$. In addition, we establish an integral operator for the series.

\section{NeCESSARY AND SUfFiCIENT CONDITIONS}

Our main characterization theorem for the class $U(\lambda, \alpha, \mu)$ is stated as Theorem 2 below.

Theorem 2. If $\xi>0$, then $\Omega(\xi, z)$ is in $U(\lambda, \alpha, \mu)$, if and only if

$$
\begin{aligned}
& \lambda \alpha \xi^{3}+(5 \lambda \alpha+\lambda-\alpha-\mu \lambda \alpha) \xi^{2}+(4 \lambda \alpha+2 \lambda-2 \alpha-2 \mu \lambda \alpha-\mu \lambda+\mu \alpha+1) \xi \\
& +(\mu-1) e^{-\xi} \leq 0 .
\end{aligned}
$$

Proof. By using the fact that

$$
\Omega(\xi, z)=z-\sum_{n=2}^{\infty} \frac{e^{-\xi} \xi^{k-1}}{(k-1) !} z^{k}
$$

and applying Lemma 1, it is adequate to show that

$$
\sum_{n=2}^{\infty}(k-\mu)[(k-1)(k \lambda \alpha+\lambda-\alpha)+1] \frac{e^{-\xi} \xi^{k-1}}{(k-1) !} \leq 1-\mu .
$$


It follows from (7) that

$$
\begin{aligned}
& \sum_{n=2}^{\infty}(k-\mu)[(k-1)(k \lambda \alpha+\lambda-\alpha)+1] \frac{e^{-\xi} \xi^{k-1}}{(k-1) !} \\
& =\sum_{n=2}^{\infty}\left\{k^{3} \lambda \alpha+k^{2}(\lambda-\alpha-\lambda \alpha-\mu \lambda \alpha)+k(\mu \lambda \alpha-\mu \lambda+\mu \alpha-\lambda+\alpha+1)\right. \\
& +\mu(\lambda-\alpha-1)\} \frac{\xi^{k-1}}{(k-1) !} .
\end{aligned}
$$

By writing

$$
\begin{gathered}
k^{3}=(k-1)(k-2)(k-3)+6(k-1)(k-2)+7(k-1)+1, \\
k^{2}=(k-1)(k-2)+3(k-1)+1
\end{gathered}
$$

and

$$
k=(k-1)+1,
$$

we obtain

$$
\begin{aligned}
& \sum_{n=2}^{\infty}(k-\mu)[(k-1)(k \lambda \alpha+\lambda-\alpha)+1] \frac{e^{-\xi} \xi^{k-1}}{(k-1) !} \\
& =\lambda \alpha \sum_{n=2}^{\infty}[(k-1)(k-2)(k-3)+6(k-1)(k-2)+7(k-1)+1] \frac{e^{-\xi} \xi^{k-1}}{(k-1) !} \\
& +(\lambda-\alpha-\lambda \alpha-\mu \lambda \alpha) \sum_{n=2}^{\infty}[(k-1)(k-2)+3(k-1)+1] \frac{e^{-\xi} \xi^{k-1}}{(k-1) !} \\
& +(\mu \lambda \alpha-\mu \lambda+\mu \alpha-\lambda+\alpha+1) \sum_{n=2}^{\infty}[(k-1)+1] \frac{e^{-\xi} \xi^{k-1}}{(k-1) !} \\
& +\mu(\lambda-\alpha-1) \sum_{n=2}^{\infty} \frac{e^{-\xi} \xi^{k-1}}{(k-1) !} \\
& =e^{-\xi}\left\{\left[e^{\xi} \xi^{3}+6 e^{\xi} \xi^{2}+7 e^{\xi} \xi+e^{\xi}-1\right] \lambda \alpha+\left[e^{\xi} \xi^{2}+3 e^{\xi} \xi+e^{\xi}-1\right](\lambda-\alpha-\lambda \alpha-\mu \lambda \alpha)\right. \\
& \left.+\left[e^{\xi} \xi+e^{\xi}-1\right](\mu \lambda \alpha-\mu \lambda+\mu \alpha-\lambda+\alpha+1)+\left(e^{\xi}-1\right) \mu(\lambda-\alpha-1)\right\} \\
& =\left[\xi^{3}+6 \xi^{2}+7 \xi+1-e^{-\xi}\right] \lambda \alpha+\left[\xi^{2}+3 \xi+1-e^{-\xi}\right](\lambda-\alpha-\lambda \alpha-\mu \lambda \alpha) \\
& +\left[\xi+1-e^{-\xi}\right](\mu \lambda \alpha-\mu \lambda+\mu \alpha-\lambda+\alpha+1)+\left(1-e^{-\xi}\right) \mu(\lambda-\alpha-1) .
\end{aligned}
$$

But, this last expression is less than or equal to $1-\mu$ if and only if (5) is satisfied. Hence the proof is completed. 
S. Porwal, Ş. Altınkaya and S. Yalçın - Poisson distribution series ...

By taking $\lambda=\alpha=0$ in Theorem 2, we state the following Corollary.

Corollary 3. If $\xi>0$, then $\Omega(\xi, z)$ is in $T^{*}(\mu)$, if and only if

$$
\xi-(1-\mu) e^{-\xi} \leq 0 .
$$

By taking $\lambda=1$ and $\alpha=0$ in Theorem 2, we state the following Corollary.

Corollary 4. If $\xi>0$, then $\Omega(\xi, z)$ is in $C(\mu)$, if and only if

$$
\xi^{2}+(3-\mu) \xi-(1-\mu) e^{-\xi} \leq 0 .
$$

\section{INCLUSION PROPERTIES}

We next explore a particular integral operator $\Lambda(\xi, z)$ as follows:

$$
\Lambda(\xi, z)=\int_{0}^{z} \frac{\Omega(\xi, t)}{t} d t
$$

Theorem 5. If $\xi>0$, then $\Lambda(\xi, z)$ defined by (8) is in $U(\lambda, \alpha, \mu)$, if and only if

$$
\begin{aligned}
& \lambda \alpha \xi^{2}+(2 \lambda \alpha+\lambda-\alpha-\mu \lambda \alpha) \xi+[1-\mu(\lambda-\alpha)]\left(1-e^{-\xi}\right) \\
& +\frac{\mu(\lambda-\alpha-1)}{\xi}\left(1-e^{-\xi}-\xi e^{-\xi}\right) \leq 1-\mu .
\end{aligned}
$$

Proof. From (8), we find

$$
\Lambda(\xi, z)=z-\sum_{n=2}^{\infty} \frac{e^{-\xi} \xi^{k-1}}{k !} z^{k} .
$$

By using Lemma 1, it is adequate to show that

$$
\sum_{n=2}^{\infty}(k-\mu)[(k-1)(k \lambda \alpha+\lambda-\alpha)+1] \frac{e^{-\xi} \xi^{k-1}}{k !} \leq 1-\mu .
$$


By virtue of the equation (10), we establish

$$
\begin{gathered}
\sum_{n=2}^{\infty}(k-\mu)[(k-1)(k \lambda \alpha+\lambda-\alpha)+1] \frac{e^{-\xi} \xi^{k-1}}{k !} \\
=\sum_{n=2}^{\infty}[(k-1)(k \lambda \alpha+\lambda-\alpha)+1] \frac{e^{-\xi} \xi^{k-1}}{(k-1) !} \\
-\mu \sum_{n=2}^{\infty}[(k-1)(k \lambda \alpha+\lambda-\alpha)+1] \frac{e^{-\xi} \xi^{k-1}}{k !} \\
=\sum_{n=2}^{\infty}(k \lambda \alpha+\lambda-\alpha) \frac{e^{-\xi} \xi^{k-1}}{(k-2) !}+\sum_{n=2}^{\infty} \frac{e^{-\xi} \xi^{k-1}}{(k-1) !} \\
-\mu \sum_{n=2}^{\infty}(k-1)(k \lambda \alpha+\lambda-\alpha) \frac{e^{-\xi} \xi^{k-1}}{k !}-\mu \sum_{n=2}^{\infty} \frac{e^{-\xi} \xi^{k-1}}{k !} \\
=\lambda \alpha \sum_{n=2}^{\infty}[(k-2)+2] \frac{e^{-\xi} \xi^{k-1}}{(k-2) !}+(\lambda-\alpha) \sum_{n=2}^{\infty} \frac{e^{-\xi} \xi^{k-1}}{(k-2) !}+\sum_{n=2}^{\infty} \frac{e^{-\xi} \xi^{k-1}}{(k-1) !} \\
-\mu \lambda \alpha \sum_{n=2}^{\infty} \frac{e^{-\xi} \xi^{k-1}}{(k-2) !}-\mu(\lambda-\alpha) \sum_{n=2}^{\infty} \frac{e^{-\xi} \xi^{k-1}}{(k-1) !}+\mu(\lambda-\alpha) \sum_{n=2}^{\infty} \frac{e^{-\xi} \xi^{k-1}}{k !}-\mu \sum_{n=2}^{\infty} \frac{e^{-\xi} \xi^{k-1}}{k !} \\
=\lambda \alpha \xi^{2} \sum_{n=2}^{\infty} \frac{e^{-\xi} \xi^{k}}{k !}+2 \lambda \alpha \xi \sum_{n=2}^{\infty} \frac{e^{-\xi} \xi^{k}}{k !}+(\lambda-\alpha) \xi \sum_{n=2}^{\infty} \frac{e^{-\xi} \xi^{k}}{k !}+\sum_{n=2}^{\infty} \frac{e^{-\xi} \xi^{k}}{k !} \\
-\mu \lambda \alpha \xi \sum_{n=2}^{\infty} \frac{e^{-\xi} \xi^{k}}{k !}-\mu(\lambda-\alpha) \sum_{n=2}^{\infty} \frac{e^{-\xi} \xi^{k}}{k !}+\frac{\mu(\lambda-\alpha)}{\xi} \sum_{n=2}^{\infty} \frac{e^{-\xi} \xi^{k}}{k !}-\frac{\mu}{\xi} \sum_{n=2}^{\infty} \frac{e^{-\xi} \xi^{k}}{k !} .
\end{gathered}
$$

Hence

$$
\begin{aligned}
= & e^{-\xi}\left[\lambda \alpha \xi^{2} e^{\xi}+(2 \lambda \alpha+\lambda-\alpha-\mu \lambda \alpha) \xi e^{\xi}+[1-\mu(\lambda-\alpha)]\left(e^{\xi}-1\right)\right. \\
& \left.+\frac{\mu(\lambda-\alpha-1)}{\xi}\left(e^{\xi}-1-\xi\right)\right] \\
= & \lambda \alpha \xi^{2}+(2 \lambda \alpha+\lambda-\alpha-\mu \lambda \alpha) \xi+[1-\mu(\lambda-\alpha)]\left(1-e^{-\xi}\right) \\
& +\frac{\mu(\lambda-\alpha+1)}{\xi}\left(1-e^{-\xi}-\xi e^{-\xi}\right) .
\end{aligned}
$$

But, this last expression is not greater than $1-\mu$ if and only if (9) is satisfied.

By taking $\lambda=\alpha=0$ in Theorem 5, we state the following Corollary.

Corollary 6. If $\xi>0$, then $\Lambda(\xi, z)$ is in $T^{*}(\mu)$, if and only if

$$
1-e^{-\xi}-\frac{\mu}{\xi}\left(1-e^{-\xi}-\xi e^{-\xi}\right) \leq 1-\mu
$$


S. Porwal, Ş. Altınkaya and S. Yalçın - Poisson distribution series ...

By taking $\lambda=1$ and $\alpha=0$ in Theorem 5, we state the following Corollary.

Corollary 7. If $\xi>0$, then $\Lambda(\xi, z)$ is in $C(\mu)$, if and only if

$$
\xi-(1-\mu) e^{-\xi} \leq 0
$$

\section{REFERENCES}

[1] Ş. Altınkaya, S. Yalçın, Poisson distribution series for analytic univalent functions, Complex Analysis and Operator Theory, (2018), 1-5, https://doi.org/10.1007/s11785-018-0764-y.

[2] Ş. Altınkaya, S. Yalçın, Poisson distribution series for certain subclasses of starlike functions with negative coefficients, Annals of Oradea University Mathematics Fascicola 2, 24, (2017), 5-8.

[3] M. Kamali, E. Kadığlu, On a new subclass of certain starlike functions with negative coefficients, Atti Sem. Mat. Fis. Univ. Modena 48, (2000), 31-44.

[4] G. Murugusundaramoorthy, K. Vijaya, S. Porwal, Some inclusion results of certain sublasses of analytic functions associated with Poisson distribution series, Hacettepe Journal of Mathematics and Statistics, DOI: 10.15672/HJMS201664513110.

[5] S. Porwal, An application of a Poisson distribution series on certain analytic functions, J. Complex Anal. 2014, (2014), 1-3, Article ID 984135.

[6] M. S. Robertson, On the theory of univalent functions, Ann. of Math. 37, (1936), 374-408.

[7] H. Silverman, Univalent functions with negative coefficients, Proc. Amer. Math. Soc. 51, (1975), 109-116.

[8] H. M. Srivastava, S. Owa, S. K. Chatterjea, A note on certain classes of starlike functions, Rend. Sem. Mat. Univ Padova 77, (1987), 115-124.

Saurabh Porwal

Department of Mathematics, UIET Campus, CSJM University, Kanpur 208024, (U.P.) India email: saurabhjcb@rediffmail.com

Şahsene Altınkaya

Department of Mathematics, Bursa Uludag University, 
S. Porwal, S. Altınkaya and S. Yalçın - Poisson distribution series ...

16059, Bursa, Turkey

email: sahsenealtinkaya@gmail.com

Sibel Yalçın

Department of Mathematics, Bursa Uludag University,

16059, Bursa, Turkey

email: syalcin@uludag.edu.tr 